\title{
Low-frequency stimulation of the external globus palladium produces anti-epileptogenic and anti- ictogenic actions in rats
}

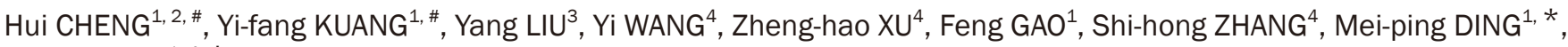 \\ Zhong $\mathrm{CHEN}^{1,4, *}$ \\ ${ }^{1}$ Department of Neurology, Epilepsy Center, Second Affiliated Hospital, School of Medicine, Zhejiang University, Hangzhou 310058, \\ China; ${ }^{2}$ Department of Neurology, Sir Run Run Shaw Hospital, School of Medicine, Zhejiang University, Hangzhou 310058, China; \\ ${ }^{3}$ Department of Neurology, Zhejiang Hospital, Hangzhou 310058, China; ${ }^{4}$ Department of Pharmacology, Key Laboratory of Medical \\ Neurobiology of the Ministry of Health of China, College of Pharmaceutical Sciences, Zhejiang University, Hangzhou 310058, China
}

Aim: To investigate the anti-epileptic effects of deep brain stimulation targeting the external globus palladium (GPe) in rats. Methods: For inducing amygdala kindling and deep brain stimulation, bipolar stainless-steel electrodes were implanted in SD rats into right basolateral amygdala and right GPe, respectively. The effects of deep brain stimulation were evaluated in the amygdala kindling model, maximal electroshock model (MES) and pentylenetetrazole (PTZ) model. Moreover, the background EEGs in the amygdala and GPe were recorded.

Results: Low-frequency stimulation $(0.1 \mathrm{~ms}, 1 \mathrm{~Hz}, 15 \mathrm{~min})$ at the GPe slowed the progression of seizure stages and shortened the after-discharge duration (ADD) during kindling acquisition. Furthermore, low-frequency stimulation significantly decreased the incidence of generalized seizures, suppressed the average stage, and shortened the cumulative ADD and generalized seizure duration in fully kindled rats. In addition, low-frequency stimulation significantly suppressed the average stage of MES-induced seizures and increased the latency to generalized seizures in the PTZ model. High-frequency stimulation (0.1 ms, $130 \mathrm{~Hz}, 5 \mathrm{~min})$ at the GPe had no anti-epileptic effect and even aggravated epileptogenesis induced by amygdala kindling. EEG analysis showed that low-frequency stimulation at the GPe reversed the increase in delta power, whereas high-frequency stimulation at the GPe had no such effect. Conclusion: Low-frequency stimulation, but not high-frequency stimulation, at the GPe exerts therapeutic effect on temporal lobe epilepsy and tonic-colonic generalized seizures, which may be due to interference with delta rhythms. The results suggest that modulation of GPe activity using low-frequency stimulation or drugs may be a promising epilepsy treatment.

Keywords: epilepsy; deep brain stimulation; external globus palladium; seizures; amygdala kindling; maximal electroshock model; pentylenetetrazole model; electroencephalogram

Acta Pharmacologica Sinica (2015) 36: 957-965; doi: 10.1038/aps.2015.45; published online 22 June 2015

\section{Introduction}

Epilepsy is a neurological disorder that affects $1 \%-2 \%$ of the population $^{[1]}$. Despite optimal medication therapy, more than $30 \%$ of patients suffer from persistent seizures ${ }^{[2]}$. A subset of these patients are candidates for open surgery, which remains limited due to anticipated functional deficits, such as memory loss or cognitive impairment ${ }^{[3]}$. Moreover,

\footnotetext{
\# These authors contributed equally to this work.

* To whom correspondence should be addressed.

E-mail chenzhong@zju.edu.cn (Zhong CHEN); meipingd@163.com (Mei-ping DING)

Received 2014-12-17 Accepted 2015-04-07
}

patients with seizures arising from the eloquent cortex or with seizures that are multiple focal, bilateral, or generalized are not candidates for resective surgery ${ }^{[4]}$. Thus, alternative therapeutic approaches and effective drug targets are needed for the remaining refractory patients.

Deep brain stimulation (DBS) is emerging as a new and promising option to treat intractable epilepsies with the advantages of reversibility, adjustability and low risk of complications ${ }^{[5,6]}$. Both clinical and animal studies have demonstrated that DBS targeting specific brain areas protects against epileptic seizures ${ }^{[7,8]}$. Recently, we reported for the first time that low frequency stimulation (LFS) of the regions outside the kindling focus, such as the central piriform $\operatorname{cortex}^{[9,10]}$, the 
cerebellar fastigial nucleus ${ }^{[11]}$, the ventral hippocampus CA3 subfield $^{[12]}$, the entorhinal cortex $(\mathrm{EC})^{[13]}$ and the subiculum ${ }^{[14]}$, inhibits amygdaloid-kindling seizures in rats. These results indicate that LFS can induce potent antiepileptic actions and that brain regions outside the epileptic focus can be used as targets of LFS. However, LFS of some brain structures may result in no effect or even in the aggravation of seizures ${ }^{[15,16]}$, which indicates that the anticonvulsant effect of DBS is highly brain region-dependent. Currently, the best target is unknown, and studies do not favor one target over another. Thus, the selection of targets and the optimal stimulation parameters should be further investigated.

External globus palladium (GPe) is composed of GABAergic cells and considered the central part in the basal ganglia (BG) circuits, which contribute to motor activity organization ${ }^{[17]}$. The GPe receives inputs from two major BG input nuclei, the neostriatum (Str) and the subthalamic nucleus (STN), and sends its output to the cerebellum via the thalamus ${ }^{[18]}$. Recently, substantial evidence has shown that BG circuits are involved in temporal lobe epilepsy ${ }^{[19]}$. The GPe, as an important part of the corticothalamic circuit, is believed to participate in seizure propagation ${ }^{[20-22]}$. For example, electroencephalography (EEG) and functional magnetic resonance imaging measurements have demonstrated that the blood oxygen level-dependent signal changes are associated with interictal discharges at the epileptic focus and the $\mathrm{BG}^{[23]}$. Disinhibition of the globus pallidium by local injection of a GABAa antagonist suppresses absence seizures, whereas inhibition of the pallidium by a GABAa agonist aggravates absence seizures ${ }^{[24]}$, indicating that GABAergic neurons of the globus pallidium play an important role in epilepsy. Moreover, regional atrophy in the bilateral globus pallidium has been found in idiopathic generalized epilepsy (GS) ${ }^{[25]}$. Therefore, the GPe may be a promising target of DBS for interfering with epilepsy.

The present study was designed to investigate the effect of different stimulation frequencies (LFS and HFS) of DBS targeted at the GPe in three independent rat seizure models. The amygdaloid-kindling model is a widely used model of temporal lobe epilepsy that mimics complex partial seizures and secondary $\mathrm{GS}^{[26]}$. The maximal electroshock seizure (MES) models are the models of generalized tonic-clonic seizures that are usually used to assess drug efficacy or other therapies ${ }^{[27]}$. In addition, the pentylenetetrazole (PTZ)-induced seizure model is used as absence seizures.

\section{Materials and methods Animals}

Male Sprague-Dawley rats (260-300 g, Grade II, Certificate № SCXK2008-0033, Experimental Animal Center, Zhejiang Academy of Medical Science, Hangzhou, China) were maintained in individual cages under a 12-h light-dark cycle (lights on 08:00-20:00) with free access to food and water. Experiments were carried out every day between 09:00 AM and 05:00 PM. All experiments were carried out in accordance with the ethical guidelines of the Zhejiang University Animal Experimentation Committee and were in complete compliance with the
National Institutes of Health Guide for the Care and Use of Laboratory Animals.

\section{Surgery}

Under pentobarbital sodium anesthesia $(45 \mathrm{mg} / \mathrm{kg}$, ip), rats were mounted in a stereotaxic apparatus (512600, Stoelting, Wood Dale, IL, USA). Electrodes were implanted into the right basolateral amygdala (AP: $-2.4 \mathrm{~mm}, \mathrm{ML}:-4.8 \mathrm{~mm}$, DV: $-8.8 \mathrm{~mm}$ ) for kindling stimulation and the right GPe (AP: $-0.8 \mathrm{~mm}$, ML: $-3.0 \mathrm{~mm}$, DV: $-6.4 \mathrm{~mm}$ ) for DBS. Reference and ground screws were placed in the skull over the cerebellum (AP: $-10.5 \mathrm{~mm}$, ML: $-1.5 \mathrm{~mm}$ ) and secured with dental cement and another screw was placed over the sensorimotor cortex (AP: $+2.0 \mathrm{~mm}$, ML: $-2.0 \mathrm{~mm}$ ). All coordinates were measured from the bregma according to the atlas of Paxinos and Watson $^{[28]}$. The electrodes were made of twisted stainless-steel Teflon-coated wires (791500, diameter $0.125 \mathrm{~mm}$, A.M. Systems, Sequim, WA, USA) insulated except for approximately $0.3 \mathrm{~mm}$ at the tip, and the maximal tip separation was approximately $0.5 \mathrm{~mm}$. The electrodes were connected to a miniature receptacle, which was attached to the skull with dental cement. After the behavioral studies, the electrode locations were histologically verified in all animals.

\section{Amygdaloid-kindling seizures}

After 7-10 d of recovery, the initial after-discharge threshold (ADT) at the right amygdala was determined (monophasic square-wave pulses, $60 \mathrm{~Hz}, 1 \mathrm{~ms} /$ pulse, 60 pulses) using a constant current stimulator (SEN-7203, SS-202J; Nihon Kohden, Tokyo, Japan). The stimulation intensity was initialized at 60 $\mu \mathrm{A}$ and was subsequently increased in steps of $20 \mu \mathrm{A}$ every 30 min until at least $5 \mathrm{~s}$ of after-discharge (AD) was elicited. The minimal intensity that produced an AD ( $5 \mathrm{~s}$ or longer) was designated as the ADT and used for daily kindling stimulation thereafter. EEGs at the amygdala and the GPe were recorded using a Neuroscan system (Compumedics, Melbourne, Australia). Seizure severity was classified according to the Racine scale ${ }^{[29]}$ : (1) facial movement, (2) head nodding, (3) unilateral forelimb clonus, (4) bilateral forelimb clonus and rearing, and (5) rearing and falling. Stages 1-3 were considered focal seizures and stages $4-5$ were considered $\mathrm{GS}^{[30]}$. When the animals exhibited three consecutive stage 5 seizures, they were regarded as fully kindled.

\section{Effects of LFS and HFS on amygdaloid-kindling acquisition}

Rats were divided into three groups matched for ADTs. Group 1 received LFS (monophasic square-wave pulses, $1 \mathrm{~Hz}$, $0.1 \mathrm{~ms} /$ pulse, $15 \mathrm{~min}$ ). Group 2 received HFS (monophasic square-wave pulses, $130 \mathrm{~Hz}, 0.1 \mathrm{~ms} /$ pulse, $5 \mathrm{~min}$ ). Group 3 (control) received sham DBS: the rats were left in the chamber and connected to the apparatus but no current was delivered. The DBS were carried out immediately after the cessation of the kindling stimulation. The DBS threshold for the GPe was $1 / 5$ of the constant that induced abnormal behavior, which was established by the appearance of increased motor and abnormal behavior ( $300 \mu \mathrm{A}$ for LFS and $20 \mu \mathrm{A}$ for HFS). The 
currents used for LFS and HFS in this study had no effect on locomotor activity, indicating the safety of the stimulation parameters (Figure S1). All groups were stimulated daily until all rats in the control group were fully kindled. The seizure stage and after-discharge duration (ADD) were recorded.

\section{Effects of LFS and HFS on amygdaloid-kindled seizures}

After the rats were fully kindled (not treated with DBS during kindling), the post-kindled ADT was determined via the same procedure used for kindling ADT, and the generalized seizure threshold (GST) was determined by increasing the current intensity in steps until a GS was elicited. Then, the rats were divided into 3 groups: Group 1 received LFS; group 2 received HFS; and group 3 (control) received sham DBS. All rats were stimulated daily with the GST as the kindling current for $10 \mathrm{~d}$. The seizure stage, latency to bilateral forelimb clonus, ADD and GS duration (GSD) were recorded. In addition, both ADT and GST were determined again on $\mathrm{d} 5$ and $\mathrm{d} 10$.

\section{Effects of LFS and HFS on MES-induced seizures}

MES $(0.2 \mathrm{~s}$ at $50 \mathrm{HZ}, 60 \mathrm{~mA})$ was delivered via ear clips using a rodent shocker (Hugo Sachs Elektronik, March-Hugstetten, Germany) and produced a GS characterized by an initial tonic extension of the fore- and hindlimbs, followed by the clonic jerking of the musculature. The motor convulsion pattern was analyzed as a measure of the seizure severity, which was assigned scores based on the extent of the spread of tonic extension ${ }^{[31]}: 0$, absence of forelimb extension; 1 , complete forelimb extension without hindlimb extension; 2 , complete forelimb extension with partial hindlimb extension; and 3, complete fore- and hindlimb extension (with hindlimbs fully extended parallel to the tail). LFS or HFS was delivered 5 min before MES stimulation and remained until the cessation of seizures.

\section{Effects of LFS and HFS on PTZ-induced seizures}

Seizures were induced using PTZ at a dose of $60 \mathrm{mg} / \mathrm{kg}$ (ip). Then, the rats were placed separately under a glass funnel, and the appearance of clonic and tonic seizures and mortality were recorded by individual observation for $30 \mathrm{~min}$. Seizure intensity was classified as follows ${ }^{[16]}$ : stage 1 : facial and ear twitching; stage 2: convulsive waves throughout the body; stage 3: myoclonic jerks, rearing; stage 4: turning over onto one side; stage 5: turning over onto the back, generalized tonicclonic seizures, or death. LFS (15 min) was delivered $5 \mathrm{~min}$ prior to PTZ injection. HFS (5 min) was delivered 3 min prior to PTZ injection. Seizure stage and the latency to the onset of myoclonic jerks were measured and analyzed statistically.

\section{EEG background activity analysis}

The background EEGs in the amygdala and GPe were recorded before daily kindling stimulation in a state of awake immobility with band-pass filters spanning DC-200 Hz and sampled at $1000 \mathrm{~Hz}$. The power spectral densities of the digitized waveforms were derived using the fast Fourier transform algorithm of the Neuroscan EEG analysis software pack- age, as previously reported ${ }^{[32]}$, and the EEG power was then calculated.

\section{Statistical analysis}

Analysis of group differences in kindling acquisition was performed using a two-way analysis of variance (ANOVA) for repeated measures followed by Tukey's $t$-test. Comparisons of the number of stimulations for each seizure stage were made using the nonparametric Mann-Whitney $U$-test. In kindled seizures, a one-way ANOVA and Tukey's $t$-test were used to calculate significance. A $\chi^{2}$ test was used to compare the incidence of GS. Group differences in MES- and PTZ-induced kindled seizures were analyzed using a one-way ANOVA followed by Tukey's $t$-test and a non-parametric test for seizure stage assessment. In the analysis of EEG background activity, a two-way ANOVA and Tukey's $t$-test were used. The data are presented as the mean \pm SEM. For all analyses, the tests were two-sided, and $P<0.05$ was considered significant.

\section{Results}

\section{Effects of LFS and HFS on amygdaloid-kindling acquisition}

LFS of the GPe applied immediately after the cessation of kindling stimulation slowed the progression of seizure stages $(P<0.001$; Figure 1A) and shortened the corresponding ADD $(P<0.01$; Figure $1 \mathrm{~B})$ compared to the control. However, HFS had no effect on the seizure stage or the ADD (Figure 1A and 1B). After 25 stimulations, $42.86 \%$ rats in the LFS group were fully kindled, whereas $88.89 \%$ rats in the HFS group and all of the rats in the control group were fully kindled $(P<0.01)$.

To further analyze the stepwise progression of kindling, we calculated the number of stimulations needed to reach and remain at each seizure stage. LFS increased the number of stimulations required to reach stages $1-3(P<0.01$ for stage 1 and 3; $P<0.001$ for stage 2; Figure $1 C$ ), which was primarily due to an increase in the number of days remaining in stages $0(P<0.01)$ and $1(P<0.05$; Figure 1D) compared to the control. However, HFS had no such effect but reduced the number of stimulations needed to reach stages $4-5$ ( $P<0.05$; Figure $1 C)$, and the number of days remaining in stage 2 was decreased $(P<0.05$; Figure 1D). Representative EEGs recorded from the right amygdala, the GPe and the sensorimotor cortex on d 15 are shown in Figure 2. LFS of the GPe shortened the corresponding ADD compared to the control, whereas HFS had no effect on the ADD. The length of the ADD in LFS group was shorter than in the HFS and control groups, but their amplitudes were similar.

\section{Effects of LFS and HFS on fully kindled seizures}

LFS of the GPe decreased the incidence of GS $(P<0.01$; Figure $3 \mathrm{~A})$, suppressed the average stage $(P<0.01$; Figure $3 \mathrm{~B})$, shortened the cumulative ADD $(P<0.05$; Figure $3 C)$ and GSD $(P<0.001$; Figure 3D), and increased the average GS latency $(P<0.05$; Figure $3 \mathrm{E})$. However, HFS had no effect on kindled seizures (Figure 3). Furthermore, when the rats were fully kindled (not treated with DBS during kindling), the postkindled ADTs were approximately $58 \%-68 \%$ of the initial 

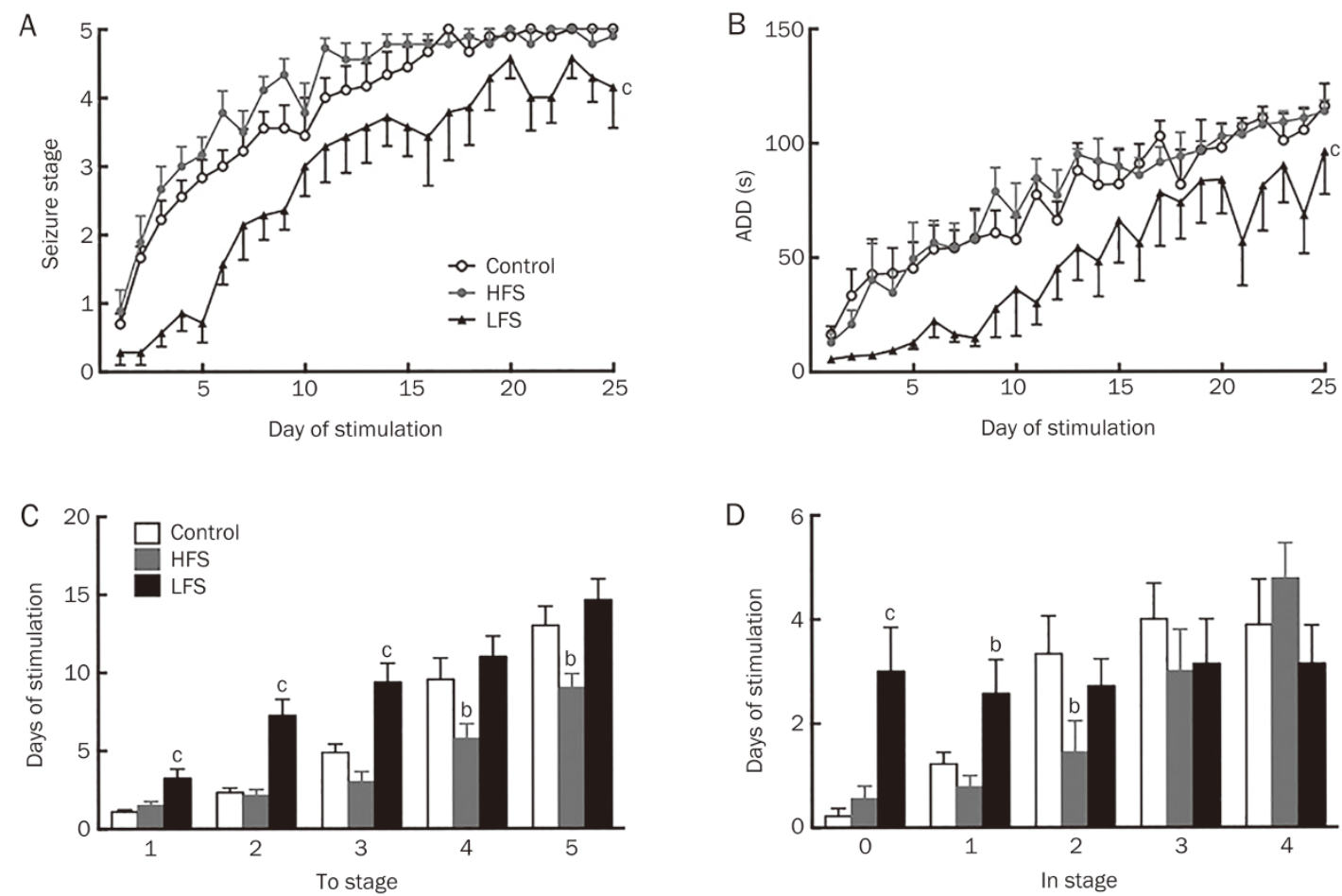

Figure 1. Effects of low/high frequency stimulation of the external globus palladium (GPe) on amygdaloid kindling acquisition. (A) Behavioral seizure stage, (B) after-discharge duration (ADD), (C) the number of stimulations required to reach each stage, and (D) the number of stimulations in each stage during kindling acquisition ( $n=8$ for the LFS group, $n=9$ for the HFS and control groups). ${ }^{b} P<0.05,{ }^{c} P<0.01$ vs the control group. A two-way repeatedmeasures ANOVA was used for the statistical analyses of A and B followed by Tukey's t-test. The non-parametric Mann-Whitney U-test was used for C and D.

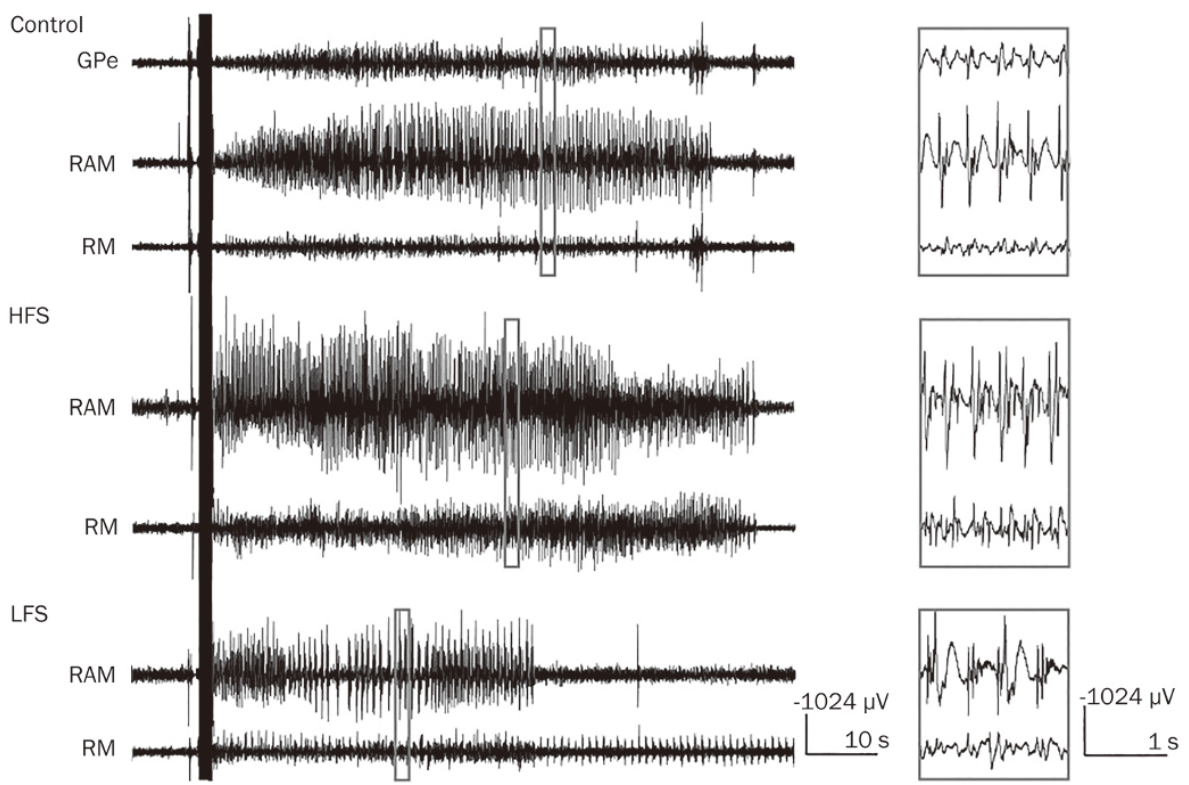

Figure 2. Representative after-discharges recorded from the right amygdala (RAM), the external globus palladium (GPe) and the sensorimotor cortex (RM) on d 15. Right panel, enlarged views of EEGs in the boxes of the left panel. The black rectangles denote kindling stimulation.

ADTs, and there were no differences among groups. On the fifth day, the ADT in the LFS group began to slightly increase (although not significant), whereas the ADT in the HFS and control groups remained unchanged. On the tenth day, only LFS increased the ADTs $(P<0.05$; Figure $3 F)$, whereas HFS did not have an effect. 


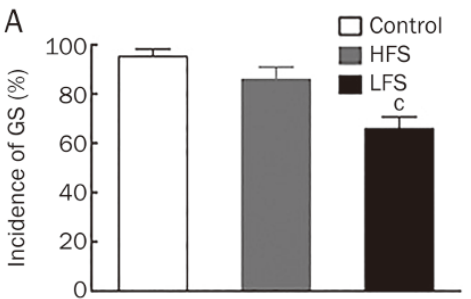

B
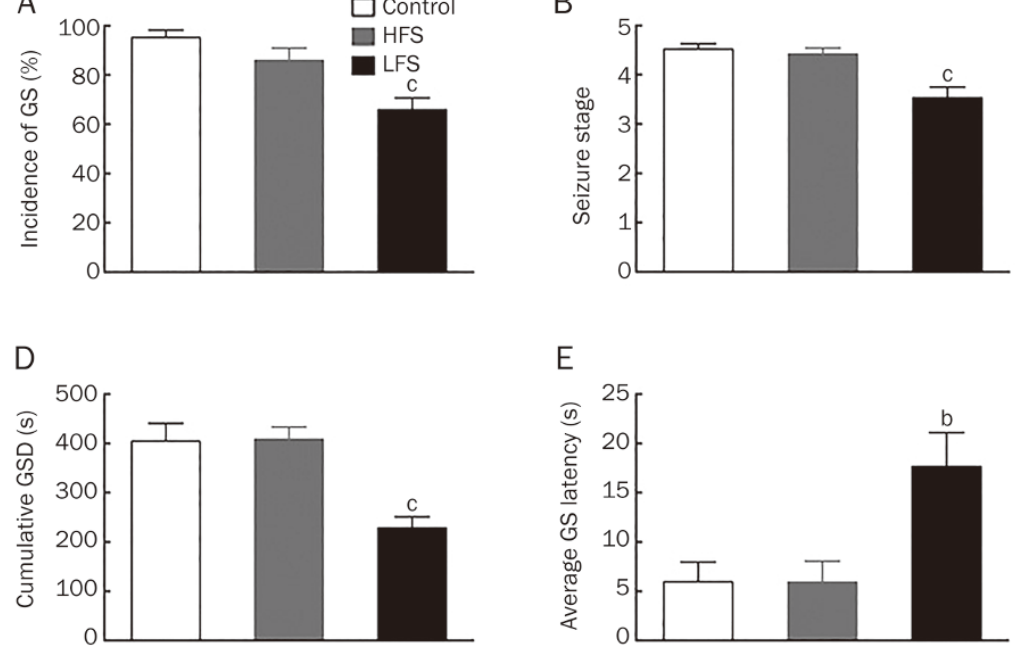

E

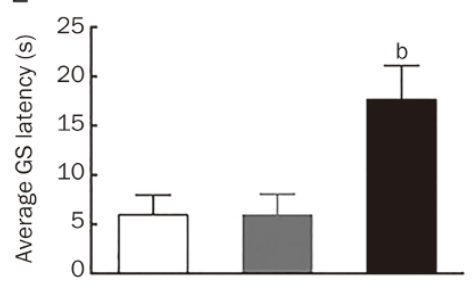

C

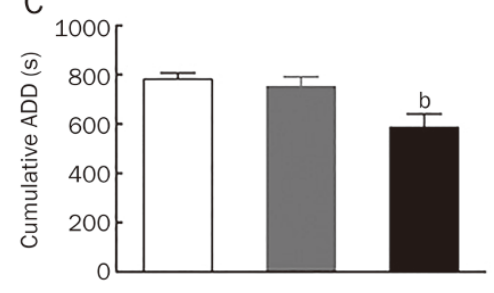

F

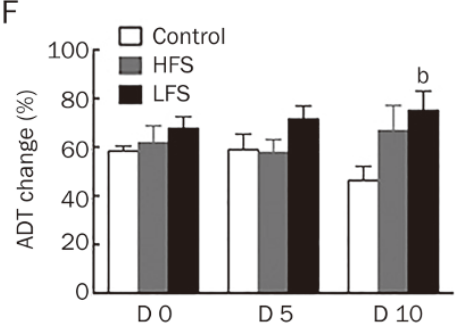

Figure 3. Effects of low/high frequency stimulation of the external globus palladium (GPe) on the fully kindled seizures. (A) Incidence of generalized seizures (GS), (B) seizure stage, (C) cumulative after-discharge duration (ADD), (D) cumulative generalized seizure duration (GSD), (E) average GS latency from d 1-4 and d 6-9, and (F) change of after-discharge threshold (ADT) tested on d 0,5 , and 10 in the fully kindled rats. ( $n=11$ for the LFS group, $n=8$ for the HFS group, and $n=6$ for the control group). ${ }^{b} P<0.05,{ }^{c} P<0.01$ compared with the control group. A chi-square test was used for $A$. A Kruskal-Wallis one-way analysis of variance followed by a Dunn's test were used for B. A one-way ANOVA followed by Tukey's $t$-test was used for C-E. A two-way ANOVA followed by Tukey's $t$-test was used for F.

\section{Effects of LFS and HFS on MES and PTZ-induced seizures}

LFS of the GPe suppressed the average seizure stage $(P<0.01$; Figure 4A) and shortened tonic hindlimb extension durations $(P<0.01$; Figure $4 C)$ in the MES model, whereas HFS had no effect. Neither LFS nor HFS exhibited a protective effect on seizure duration (Figure 4B). The average seizure stage in the LFS group was $2.00 \pm 0.53$. In contrast, the average stages in the HFS and control groups were $2.75 \pm 0.45$ and $2.80 \pm 0.42$, respectively.

In PTZ model, LFS and HFS did not exert protective effects on the seizure stages during 30-min observations, but they both prolonged the latency to myoclonic seizures $(P<0.05$, Figure 5).

\section{Effects of LFS and HFS on EEG background activity during kindling acquisition}

The analysis of the EEG background activity showed that the delta power increased during amygdala kindling acquisition, as previously reported ${ }^{[32,33]}$, which is considered an EEG marker of the severity of seizures ${ }^{[34,35]}$. Both the amygdala and the GPe in the control and HFS groups exhibited an incensement in delta power, especially in the GS state $(P<0.01$, compared with baseline). LFS but not HFS reversed the increase in the delta power during kindling acquisition $(P<0.05$, Figure 6$)$.

\section{Discussion}

Here, we found that (1) LFS of the GPe inhibited temporal lobe epilepsy in an amygdaloid-kindling and tonic-colonic GS models induced by MES and PTZ; (2) HFS only increased the latency to myoclonic seizures in the PTZ model and aggravated epileptogenesis in the kindling model; and (3) LFS but not HFS reversed the increase in the delta power. These results provided direct evidence that LFS of the GPe exerted
A

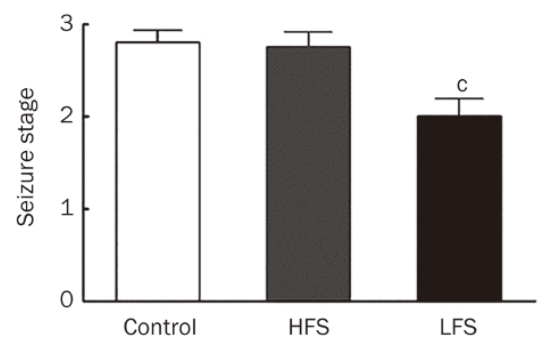

B

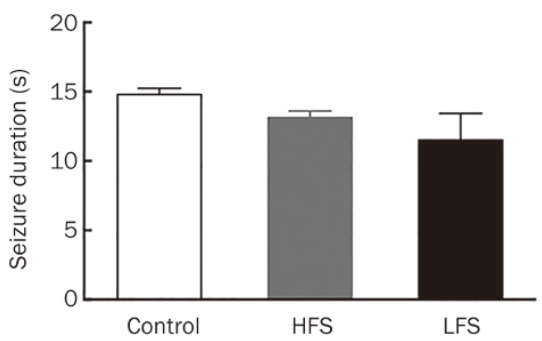

C

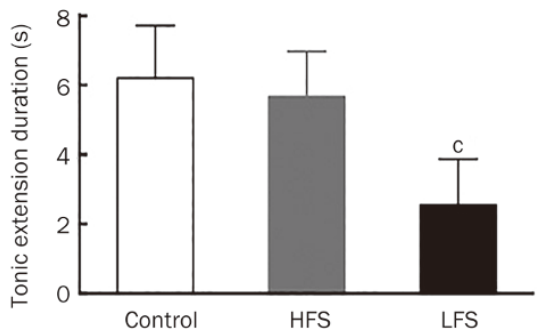

Figure 4. Effects of low/high frequency stimulation of the external globus palladium (GPe) on the maximal electroshock seizures (MES). (A) Average seizure stage, (B) seizure duration, and (C) tonic extension duration. ( $n=8$ for the LFS group, $n=8$ for the HFS and $n=10$ for the control group). ${ }^{\circ} P<0.01$ compared to the control group. A Kruskal-Wallis one-way analysis of variance followed by a Dunn's test was used for A, and a one-way ANOVA and Tukey's t-test was used for B and C. 
A

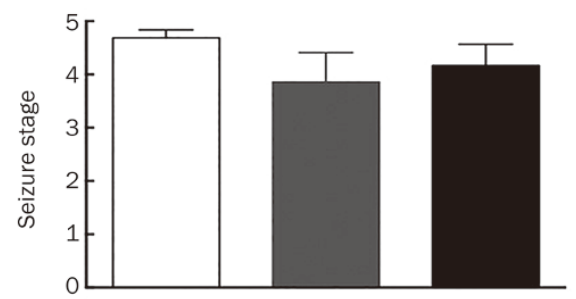

B

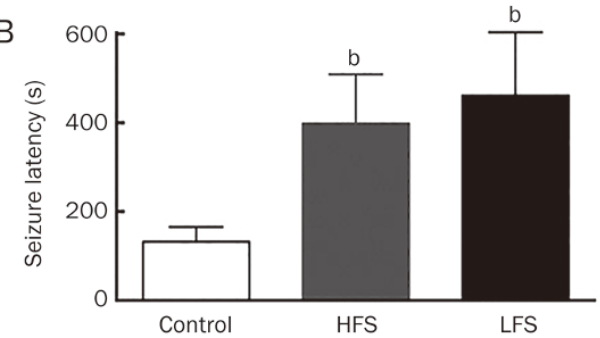

Figure 5. Effects of low/high frequency stimulation of the external globus palladium (GPe) on PTZ-induced seizures. (A) Behavioral seizure stage and (B) average latency to myoclonic jerks after PTZ injection ( $n=8$ for the control group, $n=8$ for the HFS group and $n=8$ for the LFS group). ${ }^{b} P<0.05$ compared with the control group. A one-way ANOVA followed by Tukey's $t$-test was used. therapeutic effects on epileptogenesis and GS expression, which may be due to interference with the delta rhythm, and indicated that the GPe may be a promising target for clinical LFS treatment.

Various stimulation targets for DBS have been investigated in clinical and animal studies. However, the optimal targets are yet to be determined. The success of SANTE trials ${ }^{[36]}$ (the only completed multicenter and randomized trial to date, which was conducted to demonstrate the effectiveness of bilateral HFS of the anterior thalamic nucleus (ANT) on epilepsy) demonstrated the great potential of DBS for epilepsy, but its effectiveness remains low, and research focused on other targets is required. In the present study, we found that LFS of the GPe markedly inhibited seizure stage progression and the ADD. Further analysis showed that the suppression of seizure progression was mainly due to an increase in the number of days required to reach stages 1-3 and the number of days the subject was in stages 0 and 1 . As seizures in stages 1-3 are considered focally generated in the limbic system in the amygdaloid-kindling model ${ }^{[30]}$, LFS of the GPe can retard seizure progression from focal to GS. This finding was consistent with our previous finding that LFS intervention at an early stage is critical for inhibiting epileptogenesis ${ }^{[33]}$. Because the clinical application of DBS is always initiated after epilepsy is acquired, we further determined the effects of LFS and HFS at
A
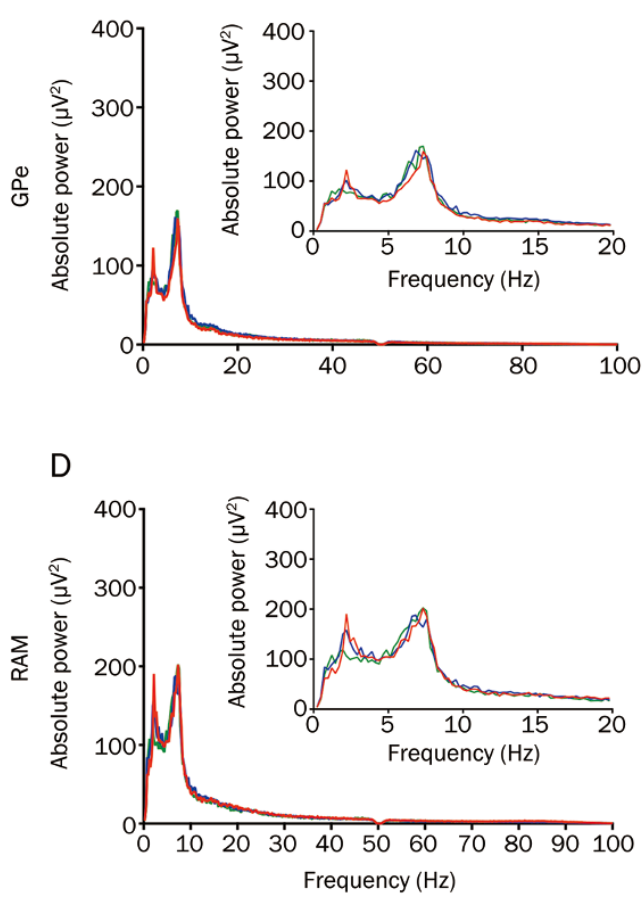

B

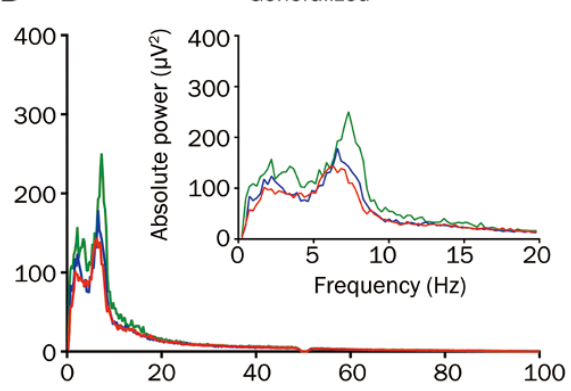

E

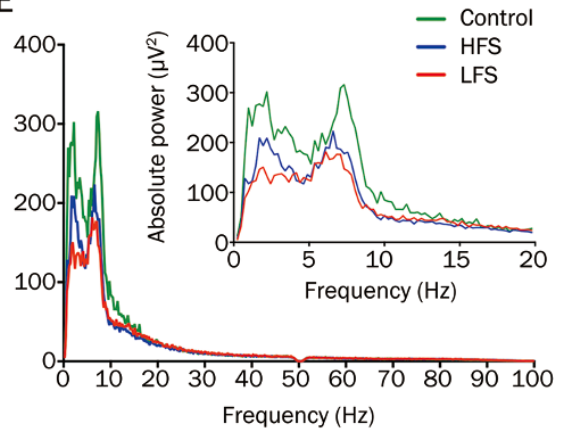

C

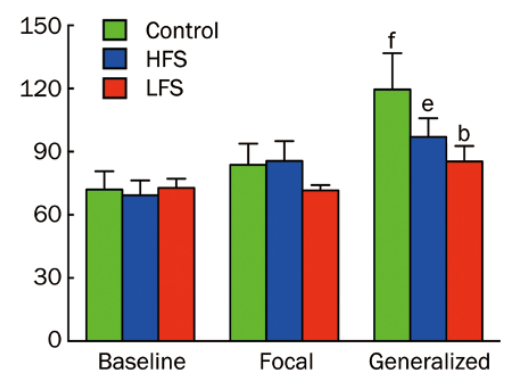

$\mathrm{F}$

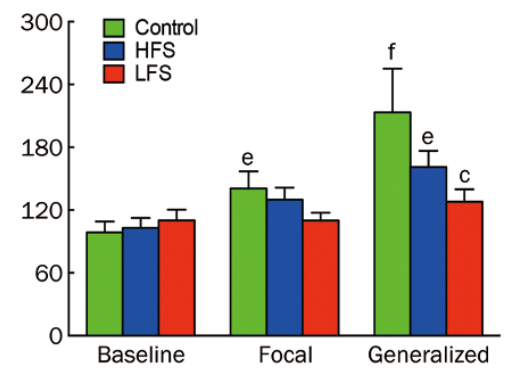

Figure 6. Effects of low/high frequency stimulation of the external globus palladium (GPe) on the background EEGs activity during kindling acquisition. Power spectra of the GPe before kindling (A) and during generalized seizures (B). Power spectra of the right amygdala (RAM) before kindling (D) and during generalized seizures $(\mathrm{E})$. Quantitative statistics of the delta power $(0-4 \mathrm{~Hz})$ at the $\mathrm{GPe}(\mathrm{C})$ and the amygdala $(\mathrm{F})$ during amygdaloid-kindling acquisition ( $n=10$ for the LFS group, $n=14$ for the HFS group, $n=10$ for the control group). ${ }^{b} P<0.05,{ }^{c} P<0.01$ compared to the control group. ${ }^{\text {e }} P<0.05$, ${ }^{f} P<0.01$ compared to baseline. A two-way repeated-measures ANOVA followed by Tukey's $t$-test was used to compare groups. 
the GPe in fully kindled rats resembling GS in the clinic and found that LFS but not HFS suppressed kindled seizures and prevented the decline of the ADT, suggesting an inhibition of the long-term susceptibility to seizures. Furthermore, LFS also exerted some protective effects on tonic-colonic seizures in the MES and PTZ models, which suggests that GPe may be a promising effective target for clinical applications of LFS to treat different subtypes of epilepsy.

GPe is widely accepted as an integrative hub for coordinating neuronal activity across BG circuits ${ }^{[17,18]}$. It is also an important part of the corticothalamic circuit, which is believed to participate in seizure propagation ${ }^{[20-22]}$, especially in absence seizures. Disinhibition of the globus pallidium via local injection of a GABAa antagonist suppresses absence seizures, whereas inhibition of the pallidium via a GABAa agonist aggravates absence seizures ${ }^{[24]}$. In the present study, we reported for the first time that DBS of the GPe exerts an anti-epileptic effect on temporal lobe epilepsy and toniccolonic GS, which indicates that GPe is also closely related to a wide variety of seizure subtypes and may be a promising drug target for the treatment of epilepsy in the future. Plausible explanations could be speculated not only from its direct interactions with the STN, the Str and the substantia nigra $(\mathrm{SN})$, which have been reported to protect against seizures ${ }^{[37-39]}$ but also from numerous well-described connections between the striatum and the basolateral amygdala and hippocampus. Therefore, it is likely that the effectiveness of LFS of the GPe may be due to modulation of its connecting circuits.

Theoretical studies have shown that targeting the GPe has several advantages. First, BG circuits modulated by the GPe stimulation most likely cannot generate seizures but act as an obstacle to the further spread of ictal activity ${ }^{[19]}$. The time course of the oscillatory activities and the absence of the epileptiform EEG activities in the BG indicate an inhibitory role of the BG in temporal lobe seizures. This "filtering effect" makes GPe DBS potentially safer. Second, GPe is a relatively large and well-defined nucleus for stereotaxy and DBS clinical trials or therapy in Parkinson's diease (PD), dystonia and Huntington's disease, making the placement of stimulating electrodes more straightforward ${ }^{[18,40,41]}$. In our experiments, the electrodes were correctly placed in the GPe in almost all rats $(125 / 128)$. In comparison, the multiple subnuclei of the ANT may be the source of variability and decreased effectiveness of DBS in the clinical trials reported thus far ${ }^{[42]}$. Recently, a study of DBS treatment for PD indicated that, compared to the STN and the GPi, the GPe may serve as an alternative target for DBS because the GPe is not complicated by the spread of current into the internal capsule that can evoke a different set of changes in the pattern and rate of neural firing throughout the pallidothalamic network ${ }^{[43]}$. These features potentially provide greater benefits and less side effects.

In addition, we found that GPe LFS has a therapeutic effect on epilepsy, whereas HFS may even aggravate epileptogenesis in the kindling model. This contrast in the effects of LFS and HFS is intriguing. Although the mechanisms underlying the therapeutic effect of DBS remain unclear, the action of HFS and LFS may be completely different. Long-term depression (LTD) or neuronal firing activity modulation has been commonly suggested as the mechanism of LFS in many reports ${ }^{[4,45]}$, whereas HFS may act via a mechanism of an EEG desynchronization or functional lesions ${ }^{[46-49]}$, which may lead to different effects of DBS targeting different brain regions. In the present study, by analyzing the EEG power, we found that LFS but not HFS reversed the increase in the delta power during kindling acquisition. The delta power is considered an EEG marker of the epileptic focus ${ }^{[34,35,50]}$ and is negatively modulated by GABAergic neurons ${ }^{[51]}$. GPe LFS may activate local GABAergic neurons to reverse the increase in the delta power, whereas HFS may induce functional lesion of GABAergic neurons, leading to the aggravation of epileptogenesis by kindling.

In conclusion, present study provides direct evidence that LFS of the GPe exerts therapeutic effects on epileptogenesis and GS expression, which may be due to interference with the delta rhythm, indicating that the GPe may be a promising and effective target for clinical LFS treatment. Moreover, the differences in the effects of HFS and LFS for targeting the GPe may open the door to further research into the mechanisms of DBS.

\section{Acknowledgements}

This work was funded by the National Natural Science Foundation of China (91332202, 81273492, 81271624, and 81201007) and the Program for Zhejiang Leading Team of S\&T Innovation (2011R50014).

\section{Author contribution}

Mei-ping DING and Zhong CHEN proposed the hypothesis, designed the experiments and wrote the manuscript; Hui CHENG, Yi-fang KUANG, Yang LIU, Yi WANG, and Zhenghao XU performed the experiments; Hui CHENG, Yi-fang KUANG, and Yi WANG analyzed the data; Feng GAO and Shi-hong ZHANG discussed the results.

\section{Supplementary information}

Supplementary information is available at Acta Pharmacologica Sinica's website.

\section{References}

1 Moshe SL, Perucca E, Ryvlin P, Tomson T. Epilepsy: new advances. Lancet 2015; 385: 884-98.

2 Löscher W, Klitgaard H, Twyman RE, Schmidt D. New avenues for anti-epileptic drug discovery and development. Nat Rev Drug Discov 2013; 12: 757-76.

3 Berg AT. Epilepsy: Efficacy of epilepsy surgery: what are the questions today? Nat Rev Neurol 2011; 7: 311-2.

4 Jette N, Wiebe S. Update on the surgical treatment of epilepsy. Curr Opin Neurol 2013; 26: 201-7.

5 Laxpati NG, Kasoff WS, Gross RE. Deep brain stimulation for the treatment of epilepsy: circuits, targets, and trials. Neurotherapeutics 2014; 11: 508-26.

6 Fisher RS, Velasco AL. Electrical brain stimulation for epilepsy. Nat Rev Neurol 2014; 10: 261-70. 
7 Lockman J, Fisher RS. Therapeutic brain stimulation for epilepsy. Neurol Clin 2009; 27: 1031-40.

8 Theodore WH, Fisher RS. Brain stimulation for epilepsy. Lancet Neurol 2004; 3: 111-8.

9 Yang LX, Jin CL, Zhu-Ge ZB, Wang S, Wei EQ, Bruce IC, et al. Unilateral low-frequency stimulation of central piriform cortex delays seizure development induced by amygdaloid kindling in rats. Neuroscience 2006; 138: 1089-96.

10 Zhu-Ge ZB, Zhu YY, Wu DC, Wang S, Liu LY, Hu WW, et al. Unilateral low-frequency stimulation of central piriform cortex inhibits amygdaloid-kindled seizures in Sprague-Dawley rats. Neuroscience 2007; 146: 901-6.

11 Wang S, Wu DC, Ding MP, Li Q, Zhuge ZB, Zhang SH, et al. Lowfrequency stimulation of cerebellar fastigial nucleus inhibits amygdaloid kindling acquisition in Sprague-Dawley rats. Neurobiol Dis 2008; 29: $52-8$.

12 Zhang SH, Sun HL, Fang Q, Zhong K, Wu DC, Wang S, et al. Lowfrequency stimulation of the hippocampal CA3 subfield is antiepileptogenic and anti-ictogenic in rat amygdaloid kindling model of epilepsy. Neurosci Lett 2009; 455: 51-5.

13 Xu ZH, Wu DC, Fang Q, Zhong K, Wang S, Sun HL, et al. Therapeutic time window of low-frequency stimulation at entorhinal cortex for amygdaloid-kindling seizures in rats. Epilepsia 2010; 51: 1861-4.

14 Zhong $\mathrm{K}$, Wu DC, Jin MM, Xu ZH, Wang Y, Hou WW, et al. Wide therapeutic time-window of low-frequency stimulation at the subiculum for temporal lobe epilepsy treatment in rats. Neurobiol Dis 2012; 48: 20-6.

15 Wang S, Wu DC, Fan XN, Zhu MZ, Hu QY, Zhou D, et al. Mediodorsal thalamic stimulation is not protective against seizures induced by amygdaloid kindling in rats. Neurosci Lett 2010; 481: 97-101.

16 Wu DC, Zhu-Ge ZB, Yu CY, Fang Q, Wang S, Jin CL, et al. Lowfrequency stimulation of the tuberomammillary nucleus facilitates electrical amygdaloid-kindling acquisition in Sprague-Dawley rats. Neurobiol Dis 2008; 32: 151-6.

17 Gittis AH, Berke JD, Bevan MD, Chan CS, Mallet N, Morrow MM, et al. New roles for the external globus pallidus in basal ganglia circuits and behavior. J Neurosci 2014; 34: 15178-83.

18 Shmuelof L, Krakauer JW. Are we ready for a natural history of motor learning? Neuron 2011; 72: 469-76.

19 Rektor I, Kuba R, Brazdil M, Chrastina J. Do the basal ganglia inhibit seizure activity in temporal lobe epilepsy? Epilepsy Behav 2012; 25 : 56-9.

20 Rektor I, Tomcik J, Mikl M, Marecek R, Brazdil M, Rektorova I. Association between the basal ganglia and large-scale brain networks in epilepsy. Brain Topogr 2013; 26: 355-62.

21 Zhang Z, Liao W, Bernhardt B, Wang Z, Sun K, Yang F, et al. Brain iron redistribution in mesial temporal lobe epilepsy: a susceptibilityweighted magnetic resonance imaging study. BMC Neurosci 2014; 15: 117.

22 Plantone D, Renna R, Grossi D, Plantone F, lorio R. Teaching NeuroImages: Basal ganglia involvement in facio-brachial dystonic seizures associated with LGI1 antibodies. Neurology 2013; 80: e183-4.

23 Federico P, Archer JS, Abbott DF, Jackson GD. Cortical/subcortical BOLD changes associated with epileptic discharges: an EEG-fMRI study at 3 T. Neurology 2005; 64: 1125-30.

24 Deransart C, Riban V, Le BT, Hechler V, Marescaux C, Depaulis A. Evidence for the involvement of the pallidum in the modulation of seizures in a genetic model of absence epilepsy in the rat. Neurosci Lett 1999; 265: 131-4.

25 Du H, Zhang Y, Xie B, Wu N, Wu G, Wang J, et al. Regional atrophy of the basal ganglia and thalamus in idiopathic generalized epilepsy. J
Magn Reson Imaging 2011; 33: 817-21.

26 Morimoto K, Fahnestock M, Racine RJ. Kindling and status epilepticus models of epilepsy: rewiring the brain. Prog Neurobiol 2004; 73: 1-60.

27 Bialer M, White HS. Key factors in the discovery and development of new antiepileptic drugs. Nat Rev Drug Discov 2010; 9: 68-82.

28 Paxinos G, Watson C. The rat brain in stereotaxic coordinates. 6th ed. Amsterdam; Boston; Academic Press/Elsevier; 2007.

29 Racine RJ. Modification of seizure activity by electrical stimulation. II. Motor seizure. Electroencephalogr Clin Neurophysiol 1972; 32: 281-94.

30 Sato M, Racine RJ, McIntyre DC. Kindling: basic mechanisms and clinical validity. Electroencephalogr Clin Neurophysiol 1990; 76: 459-72.

31 Tortella FC, Long JB, Holaday JW. Endogenous opioid systems: physiological role in the self-limitation of seizures. Brain Res 1985; 332: 174-8.

32 Xu Z, Wang Y, Jin M, Yue J, Xu C, Ying X, et al. Polarity-dependent effect of low-frequency stimulation on amygdaloid kindling in rats. Brain Stimul 2013; 6: 190-7.

33 Wang Y, Xu Z, Cheng H, Guo Y, Xu C, Wang S, et al. Low-frequency stimulation inhibits epileptogenesis by modulating the early network of the limbic system as evaluated in amygdala kindling model. Brain Struct Funct 2014; 219: 1685-96.

34 Gambardella A, Gotman J, Cendes F, Andermann F. Focal intermittent delta activity in patients with mesiotemporal atrophy: a reliable marker of the epileptogenic focus. Epilepsia 1995; 36: 122-9.

35 Geyer JD, Bilir E, Faught RE, Kuzniecky R, Gilliam F. Significance of interictal temporal lobe delta activity for localization of the primary epileptogenic region. Neurology 1999; 52: 202-5.

36 Fisher R, Salanova V, Witt T, Worth R, Henry T, Gross R, et al. Electrical stimulation of the anterior nucleus of thalamus for treatment of refractory epilepsy. Epilepsia 2010; 51: 899-908.

37 Vercueil L, Benazzouz A, Deransart C, Bressand K, Marescaux C, Depaulis A, et al. High-frequency stimulation of the subthalamic nucleus suppresses absence seizures in the rat: comparison with neurotoxic lesions. Epilepsy Res 1998; 31: 39-46.

38 Deransart C, Le BT, Marescaux C, Depaulis A. Role of the subthalamonigral input in the control of amygdala-kindled seizures in the rat. Brain Res 1998; 807: 78-83.

39 Depaulis A, Vergnes M, Marescaux C. Endogenous control of epilepsy: the nigral inhibitory system. Prog Neurobiol 1994; 42: 33-52.

40 Vitek JL, Hashimoto T, Peoples J, DeLong MR, Bakay RA. Acute stimulation in the external segment of the globus pallidus improves parkinsonian motor signs. Mov Disord 2004; 19: 907-15.

41 Ligot N, Krystkowiak P, Simonin C, Goldman S, Peigneux P, Van Naemen J, et al. External globus pallidus stimulation modulates brain connectivity in Huntington's disease. J Cereb Blood Flow Metab 2011; 31: 41-6.

42 Lega BC, Halpern CH, Jaggi JL, Baltuch GH. Deep brain stimulation in the treatment of refractory epilepsy: update on current data and future directions. Neurobiol Dis 2010; 38: 354-60.

43 Vitek JL, Zhang J, Hashimoto T, Russo GS, Baker KB. External pallidal stimulation improves parkinsonian motor signs and modulates neuronal activity throughout the basal ganglia thalamic network. Exp Neurol 2012; 233: 581-6.

44 Goodman JH, Berger RE, Tcheng TK. Preemptive low-frequency stimulation decreases the incidence of amygdala-kindled seizures. Epilepsia 2005; 46: 1-7.

45 Fujii S, Saito K, Miyakawa H, Ito K, Kato H. Reversal of long-term potentiation (depotentiation) induced by tetanus stimulation of the 
input to CA1 neurons of guinea pig hippocampal slices. Brain Res 1991; 555: 112-22.

46 Lozano AM, Lipsman N. Probing and regulating dysfunctional circuits using deep brain stimulation. Neuron 2013; 77: 406-24.

47 McIntyre CC, Savasta M, Kerkerian-Le Goff L, Vitek JL. Uncovering the mechanism(s) of action of deep brain stimulation: activation, inhibition, or both. Clin Neurophysiol 2004; 115: 1239-48.

48 Dostrovsky JO, Levy R, Wu JP, Hutchison WD, Tasker RR, Lozano AM. Microstimulation-induced inhibition of neuronal firing in human globus pallidus. J Neurophysiol 2000; 84: 570-4.
49 Hashimoto T, Elder CM, Okun MS, Patrick SK, Vitek JL. Stimulation of the subthalamic nucleus changes the firing pattern of pallidal neurons. J Neurosci 2003; 23: 1916-23.

50 Reiher J, Beaudry M, Leduc CP. Temporal intermittent rhythmic delta activity (TIRDA) in the diagnosis of complex partial epilepsy: sensitivity, specificity and predictive value. Can J Neurol Sci 1989; 16: 398-401.

51 Carracedo LM, Kjeldsen H, Cunnington L, Jenkins A, Schofield I, Cunningham MO, et al. A neocortical delta rhythm facilitates reciprocal interlaminar interactions via nested theta rhythms. J Neurosci 2013; 33: 10750-61. 